\title{
Erratum
}

\section{Self-renewal versus differentiation, a job for the mighty morphogens}

Leonard I. Zon

Nature Immunology 2, 142-143 (2001).

E In the February issue of Nature Immunology some text was printed incorrectly. On page 143, column 1, line 13 of paragraph 2 should read "The effects of Shh are exerted by establishing BMP homeostasis in the bone marrow; Shh inhibits both BMP-4 and a BMP inhibitor called Noggin.”. In addition, line 34 of the same paragraph should read "Thus, for blood cells in the bone marrow, the regulation of BMP and Noggin by Shh "fine-tunes" the local effective concentration of BMP.".

\section{Correction}

\section{Redirecting T cell specificity by TCR gene transfer}

\section{Paul A. H. Moss}

Nature Immunology 2, 900-901 (2001).

In the October issue of Nature Immunology, it should be noted that the optimal MDM2 cytotoxic epitope was amino acids 81-88, as shown in the figure, rather than amino acids $80-88$, as mentioned in the text. 